\title{
Chemical changes on, and through, the bacterial envelope in $E$. coli mutants exhibiting impaired plasmid transfer identified using time-of-flight secondary ion mass spectrometry
}

Kelly Dimovska Nilsson ${ }^{1}$, Martin Palm, ${ }^{1,2}$ James Hood, ${ }^{3}$ Jake Sheriff, ${ }^{3}$ Anne Farewell ${ }^{1,2^{*}}$, John S. Fletcher ${ }^{1,2 *}$

1. Department of Chemistry and Molecular Biology, University of Gothenburg, Gothenburg, Sweden

2. Centre for Antibiotic Resistance Research, University of Gothenburg, Gothenburg, Sweden

3. School of Engineering, Newcastle University, Newcastle upon Tyne, United Kingdom

ABSTRACT: Time-of-flight secondary ion mass spectrometry (ToF-SIMS) using a $\left(\mathrm{CO}_{2}\right)_{6 \mathrm{k}}{ }^{+}$gas cluster ion beam (GCIB) was used to analyze $E$. coli mutants previously identified as having impaired plasmid transfer capability. The particular sub-set of mutants were selected as the mutations were expected to result in changes in the bacterial envelope composition, through the deletion of genes encoding for FabF, DapF and Lpp, where the surface sensitivity of ToF-SIMS can be most useful. Analysis of arrays of spotted bacteria allowed changes in the lipid composition of the bacteria to be elucidated using multivariate analysis and confirmed through imaging of individual ion signals. Significant changes in chemical composition were observed, including a surprising loss of cyclopropanated fatty acids in the fabF mutant where FabF is associated with the elongation of $F A(16: 1)$ to $F A(18: 1)$ and not cyclopropane formation. The ability of the GCIB to generate increased higher mass signals from biological samples allowed intact lipid $A(\mathrm{~m} / \mathrm{z} 1796)$ to be detected on the bacteria and, despite a $40 \mathrm{keV}$ impact energy, depth profiled through the bacterial envelope along with other high mass ions including species at $\mathrm{m} / \mathrm{z} 1820$ and 2428 , attributed to $E C A_{C Y C}$, that were only observed below the surface of the bacteria and were notably absent in the depth profile of the Ipp mutant. The analysis provides new insights into the action of the specific pathways targeted in this study and paves the way for whole new avenues for the characterization of intact molecules within the bacterial envelope. 


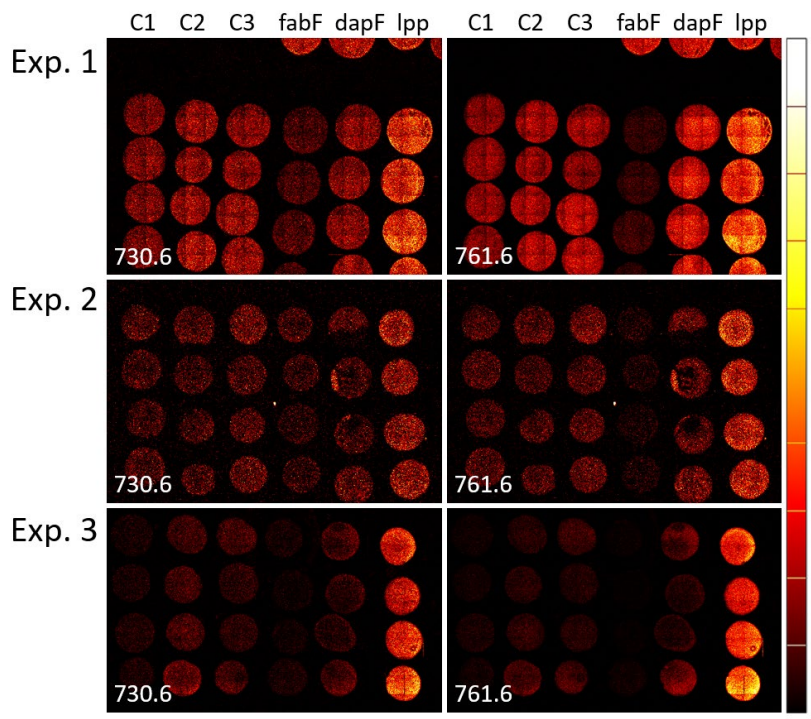

Supplementary Figure 1. a: Single ion images of species previously reported as PE(16:0)/(cp19:0) and PG(16:0)/(cp19:0) at $\mathrm{m} / \mathrm{z} 730.6(16 \mathrm{ppm})$ and $\mathrm{m} / \mathrm{z} 761.6(12 \mathrm{ppm})$ respectively.

\section{Cardiolipins}

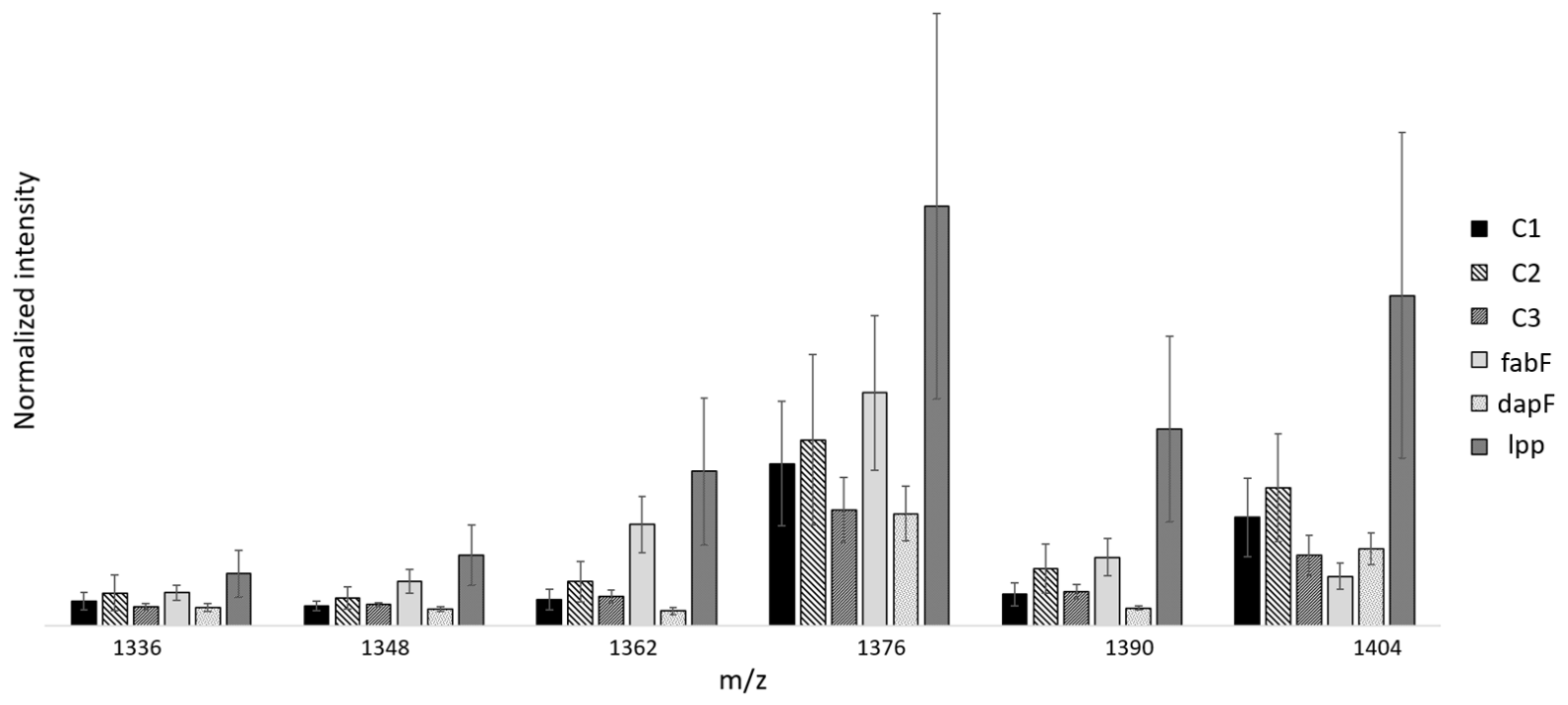

Supplementary Figure 2. Bar graph showing the average normalized intensity of controls and mutants for $m / z 1336,1348$, $1362,1376,1390$ and 1404. Error bars are of the standard error of the mean (SEM) $n=3$. 


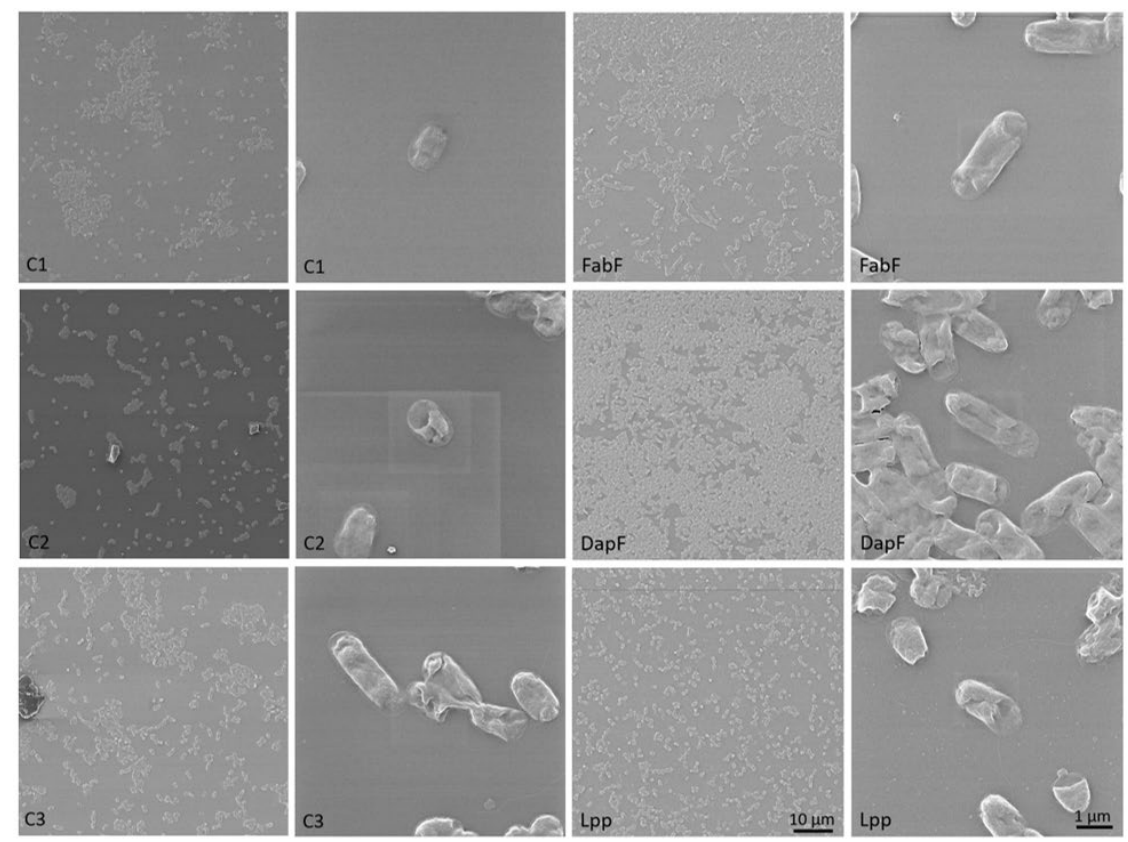

Supplementary Figure 3. HIM images of the three mutants and control bacteria at low and high magnification. Scale bars = 10 and $1 \mu \mathrm{m}$ respectively. Images were taken using a $25 \mathrm{keV}$ He primary ion beam, with beam current of $0.2 \mathrm{pA}$, using a scan dwell time of $10 \mu$ s per pixel, averaging 16 scans per image, no charge compensation was required as the samples were platinum coated. 
C1

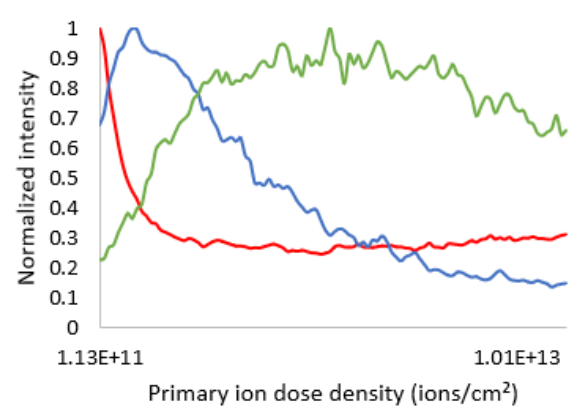

C3

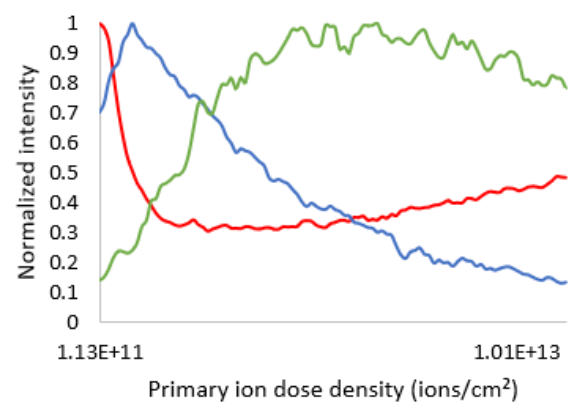

dapF

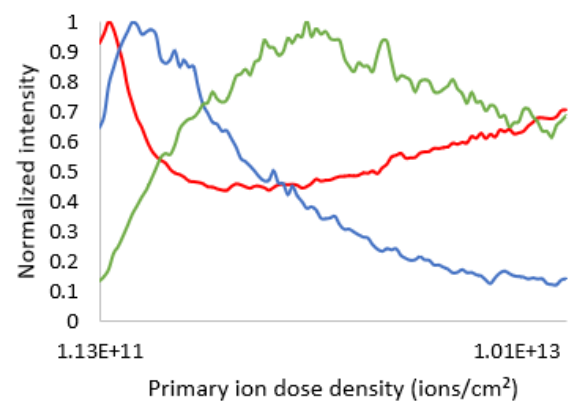

C2

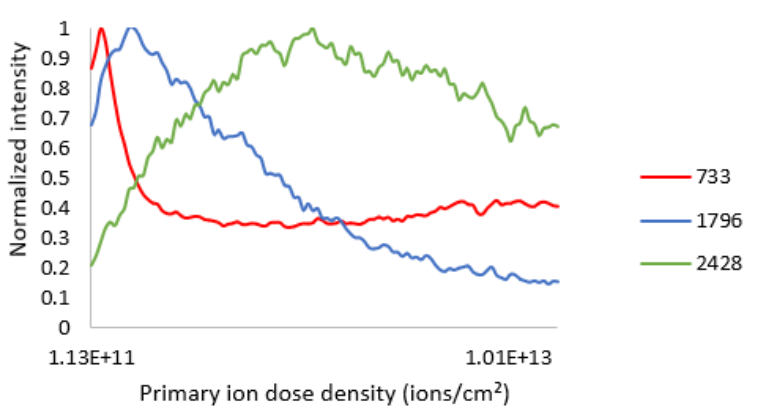

fabF

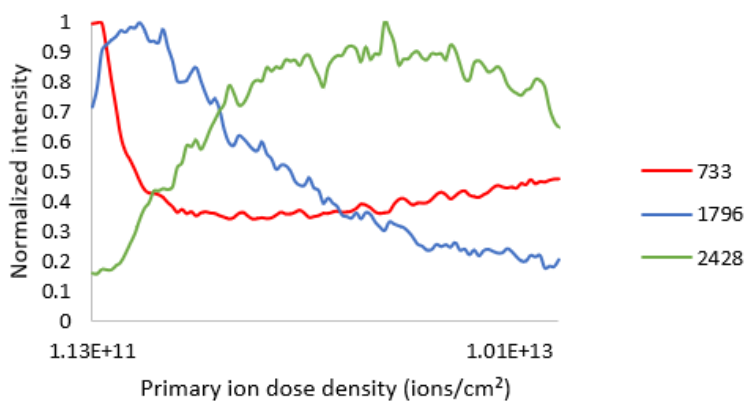

Ipp

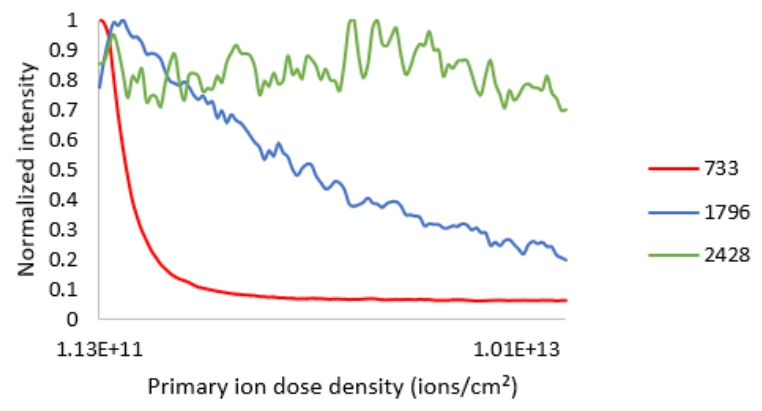

Supplementary Figure 4. Depth profiles of PG(cp33:0) at $m / z 733(\Delta 5.2 \mathrm{ppm})$, lipid A at $\mathrm{m} / z 1796(\Delta 11 \mathrm{ppm})$ and ECACYC at $m / z 2428$ (41 ppm) showing the different signal profiles of these species. Signal normalized to the highest signal detection. 